\title{
Research advances in the study of Campylobacter, Helicobacter, and Related Organisms
}

\author{
D. Scott Merrell ${ }^{1 *}$ and Alain Stintzi ${ }^{2 *}$ \\ 1 Department of Microbiology and Immunology, Uniformed Services University, Bethesda, MD, USA \\ 2 Department of Biochemistry, Microbiology and Immunology, University of Ottawa, Ottawa, ON, Canada \\ *Correspondence: douglas.merrell@usuhs.edu; astintzi@uottawa.ca
}

Edited by:

Yousef Abu Kwaik, University of Louisville School of Medicine, USA

Reviewed by:

Yousef Abu Kwaik, University of Louisville School of Medicine, USA

Campylobacter spp. and Helicobacter spp. are gastrointestinal pathogens that remain a major cause of acute gastroenteritis and gastric disease, respectively. The 16th International Workshop on Campylobacter, Helicobacter and Related Organisms (CHRO) was organized by Erin Gaynor and Christine Szymanski and was held in Vancouver, BC, Canada from August 28 to September 1, 2011. This meeting highlighted recent advances in our understanding of the epidemiology, survival mechanisms, host response, and pathogenesis of these important species. This Research Topic issue highlights each of these topics and attempts to shed insight into our growing understanding of the process of host-pathogen interactions as it relates to Campylobacter and Helicobacter. We wish to dedicate this Research Topic to Dr. Andre Dubois, who passed away unexpectedly on June 30, 2012. We are honored that one of his research articles appears in the issue and note that his loss is deeply felt by our community.

A substantive overview of the CHRO meeting is provided by Drs. Gaynor and Szymanski (2012) and the articles within this Research Topic are broadly divided into those dealing with Campylobacter and then Helicobacter. Those articles related to Helicobacter can broadly be divided into three major topics: those that address $H$. pylori lifestyle and biological processes, those that address $H$. pylori virulence factors, and those that address interaction of $H$. pylori with the host.

Within the H. pylori lifestyle and biological processes, studies from the Dubois lab investigate the mechanism by which H. pylori enters host cells (Liu et al., 2012). Though primarily considered an extracellular pathogen, it is clear that a fraction of $H$. pylori cells enter and survive within the host cell. The Dubois team presents evidence that NudA is important in this process. Next, Liechti and Goldberg review the process of membrane biogenesis in $H$. pylori and compare it to the processes that have been elucidated in Escherichia coli and Neisseria meningitidis; not surprisingly, $H$. pylori often does not follow the paradigms established in these model systems (Liechti and Goldberg, 2012). Finally, Pernitzsch and Sharma discuss transcriptome complexity and riboregulation in $\mathrm{H}$. pylori (Pernitzsch and Sharma, 2012). It has only recently become evident that H. pylori employs post-transcriptional regulation and small regulatory RNAs (sRNAs) as a mechanism of gene regulation. This fact directly conflicts the previous dogma that life within the singular niche of the host stomach has led to $H$. pylori's loss of complex gene regulation.
Among H. pylori virulence factors, CagA, and VacA are undoubtedly the best studied. As such, a number of articles are devoted to these important factors. The Solnick group presents evidence that expression of genes on the H. pylori cag pathogenicity island, which encodes for CagA, varies significantly, and that the organization of the genes into transcriptional units is conserved among several $H$. pylori strains (Ta et al., 2012). In terms of CagA delivery, the Backert group investigates sequences/domains within the CagL protein that are important for interaction with integrins and subsequent injection of CagA into host cells (Conradi et al., 2012). Next, the Guillemin group describes the utilization of a novel Drosophila system to identify host components that affect CagA activity within host cells (Reid et al., 2012). Finally, Kim and Blanke review the role of the VacA toxin in modulation of the gastric epithelium and discuss the understudied area of VacA and CagA interaction (Kim and Blanke, 2012).

The final major topic addressed among the $H$. pylori manuscripts is the consequences of $H$. pylori-host cell interaction. From the bacterial perspective, the Cover group specifically discuss what we currently know about how host cell contact alters H. pylori cells (Johnson et al., 2012). Conversely, Noto and Peek discuss our current understanding of the role of microRNAs on the process of $H$. pylori pathogenesis and gastric carcinogenesis (Noto and Peek, 2012). Finally, the Müller group tackles an intriguing question that is coming to the forefront of the H. pylori field; does colonization with $H$. pylori actually provide any benefits to the host? Recent studies suggest that this is indeed the case, and Müller et al. review how $H$. pylori immunomodulation can confer protection against allergic and chronic inflammatory disorders (Arnold et al., 2012).

Those articles related to Campylobacter can be divided into five major topics: the function of glycans and capsule polysaccharides in Campylobacter virulence and biology, the mechanism of cell invasion, Campylobacter antimicrobial resistance, molecular typing methods, and general Campylobacter metabolism and biology.

Capsular polysaccharides (CPS) protect microbes from environmental insults and host immune defenses. Guerry et al. review our current knowledge on the role of CPS in Campylobacter virulence and provide an interesting perspective on the potential of CPS as a conjugate vaccine (Guerry et al., 2012). In an original research article, Sorensen et al. demonstrate that the $O$-methyl phosphoramidate (MeOPN) moiety of the C. jejuni 
CPS is recognized as a receptor by several different phages (Sorensen et al., 2012). Interestingly, they observed in vivo phase variation of the capsular structure leading to phage resistance and suggesting phage-host co-evolution. Day et al. highlight the key role of $C$. jejuni surface glycans in its interaction with the host and the function of the host glycoconjugates in the defense against $C$. jejuni infection (Day et al., 2012).

Epithelial cell invasion is thought to be a major determinant of C. jejuni virulence. One review article and two original research papers focus on this important virulence trait (Boehm et al., 2011; Croinin and Backert, 2012; Neal-McKinney and Konkel, 2012). Boehm et al. used knockout cell lines to characterize the host signaling cascades involved in the process of $C$. jejuni invasion (Boehm et al., 2011). This study provides clear evidence of a role for the fibronectin, integrin beta1, FAK, and DOCK180/Tiam1 signaling cascade and for Racl GTPase activation in C. jejuni epithelial cell invasion. Neal-McKinney and Konkel demonstrate that the flagellum secreted protein $\mathrm{CiaC}$ is delivered into the cytosol of the host cells and interact with the cell signaling pathways involved in C. jejuni cell invasion (Neal-McKinney and Konkel, 2012), possibly the signaling cascade identified by Boehm et al.

Colonization of the host gastrointestinal tract is intimately linked to the ability to survive host defenses. In this regard Hoang et al. describe the identification of genetic loci enabling C. jejuni to resist fowlicidin-1 exposure, a potent chicken antimicrobial peptide (Hoang et al., 2012). Intriguingly, fluoroquinoloneresistant Campylobacter have been previously shown to exhibit enhanced in vivo fitness. In an original research article, Han and collaborators now confirm the role of the Thr-86-Ile mutation in gyrA in conferring fluoroquinolone-resistance and demonstrate that this mutation modulates fitness in vivo and DNA supercoiling homeostasis in Campylobacter (Han et al., 2012). Given the importance of DNA supercoiling in gene expression, Han and collaborators argue that the altered DNA supercoiling observed in the fluoroquinolone-resistant Campylobacter might be directly linked to its increased fitness. Clearly, the current increase in the prevalence of fluoroquinolone-resistant Campylobacter threatens clinical treatments. This concern prompts Dufour et al. to assess the potential of phytochemicals as preservative to reduce Campylobacter load in food (Dufour et al., 2012). This work reports the antimicrobial properties of isothiocyanates (ITC) against 24 isolates of C. jejuni demonstrating their bactericidal effects and highlights the role of the $\gamma$-glutamyl-transpeptidase gene in Campylobacter resistance to ITC.

\section{REFERENCES}

Alemka, A., Corcionivoschi, N., and Bourke, B. (2012). Defense and adaptation: the complex inter-relationship between Campylobacter jejuni and mucus. Front. Cell. Inf. Microbio. 2:15. doi: 10.3389/fcimb.2012.00015

Arnold, I. C., Hitzler, I., and Muller, A. (2012). The immunomodulatory properties of Helicobacter pylori confer protection against allergic and chronic inflammatory disorders. Front. Cell. Inf. Microbio. 2:10. doi: 10.3389/fcimb.2012.00010 Boehm, M., Krause-Gruszczynska, M., Rohde, M., Tegtmeyer, N., Takahashi, S., Oyarzabal, O. A., et al. (2011). Major host factors involved in epithelial cell invasion

The detection of Campylobacter outbreaks is hampered by the lack of defined and standardized methods to unambiguously detect and track sources of Campylobacter. Two research articles tackle this important epidemiological issue. Through the use of publically available whole genome sequences of C. jejuni and C. coli, Carrillo and collaborators describe the development of a framework to assess the performance of the existing and emerging molecular typing methods (Carrillo et al., 2012). In terms of the multilocus sequence typing methods (MLST), Miller and collaborators describe and assess the performance of four MLST methods for differentiating strains of the emerging Campylobacter species C. hyointestinalis, C. lanienae, C. sputorum, C. concisus, and C. curvus (Miller et al., 2012).

Finally, one original research article and four reviews highlight the recent developments in our understanding of Campylobacter physiology and biology. Haddad et al. describe the pleiotropic role of the polynucleotide phosphorylase (PNPase) in C. jejuni biology which affects motility, cell invasion and adherence, and chick gut colonization (Haddad et al., 2012; Matos et al., 2012). Plummer reviews our current knowledge on quorum-sensing in Campylobacter and its role in pathogenesis (Plummer, 2012). Alemka and collaborators describe Campylobacter physiology at the mucosa-luminal interface and specifically the complex interplay between C. jejuni and mucus (Alemka et al., 2012). Stahl et al. summarize Campylobacter ability to acquire and metabolize nutrients including a discussion of L-fucose metabolism in C. jejuni (Stahl et al., 2012). And lastly, Kaakoush and Mitchell highlight the emerging role of Campylobacter concisus as a new player of acute and chronic intestinal disease (Kaakoush and Mitchell, 2012).

Clearly we have learned a significant amount about Campylobacter and Helicobacter over the course of the last decades. However, as stated by Gaynor and Szymanski "both pioneers and new investigators in the CHRO research field continue to obtain 'unexpected results' demonstrating that Campylobacters and Helicobacters do not follow classic paradigms of other wellcharacterized gastrointestinal pathogens and we are learning that there is a plethora of interesting related organisms beyond C. jejuni and H. pylori." Therefore, though we have learnt much, it is clear that the coming years offer many places to advance our understanding of these important pathogens.

\section{ACKNOWLEDGMENTS}

Alain Stintzi is supported by CIHR and D. Scott Merrell is supported by the NIH and DOD. The contents are solely the responsibility of the authors and do not necessarily represent the official views of the funding agencies.

of Campylobacter jejuni: role of fibronectin, integrin betal, FAK, Tiam-1, and DOCK180 in activating Rho GTPase Racl. Front. Cell. Inf. Microbio. 1:17. doi: 10.3389/fcimb.2011.00017

Carrillo, C. D., Kruczkiewicz, P., Mutschall, S., Tudor, A., Clark, C., and Taboada, E. N. (2012). A framework for assessing the concordance of molecular typing methods and the true strain phylogeny of Campylobacter jejuni and C. coli using draft genome sequence data. Front. Cell. Inf. Microbio. 2:57. doi: 10.3389/fcimb.2012.00057

Conradi, J., Tegtmeyer, N., Wozna, M., Wissbrock, M., Michalek, C., Gagell, C., et al. (2012). An RGD helper sequence in $\mathrm{CagL}$ 
of Helicobacter pylori assists in interactions with integrins and injection of CagA. Front. Cell. Inf. Microbio. 2:70. doi: 10.3389/fcimb. 2012.00070

Day, C. J., Semchenko, E. A., and Korolik, V. (2012). Glycoconjugates play a key role in Campylobacter jejuni infection: interactions between host and pathogen. Front. Cell. Inf. Microbio. 2:9. doi: 10.3389/fcimb.2012.00009

Dufour, V., Alazzam, B., Ermel, G., Thepaut, M., Rossero, A., Tresse, O., et al. (2012). Antimicrobial activities of isothiocyanates against Campylobacter jejuni isolates. Front. Cell. Inf. Microbio. 2:53. doi: 10.3389/fcimb.2012.00053

Gaynor, E. C., and Szymanski, C. M. (2012). The 30(th) anniversary of Campylobacter, Helicobacter, and related organisms workshops-what have we learned in three decades? Front. Cell. Inf. Microbio. 2:20. doi: 10.3389/fcimb.2012.00020

Guerry, P., Poly, F., Riddle, M., Maue, A. C., Chen, Y. H., and Monteiro, M. A. (2012). Campylobacter polysaccharide capsules: virulence and vaccines. Front. Cell. Inf. Microbio. 2:7. doi: 10.3389/fcimb. 2012.00007

Haddad, N., Tresse, O., Rivoal, K., Chevret, D., Nonglaton, Q., Burns, C. M., et al. (2012). Polynucleotide phosphorylase has an impact on cell biology of Campylobacter jejuni. Front. Cell. Inf. Microbio. 2:30. doi: 10.3389/fcimb.2012.00030

Han, J., Wang, Y., Sahin, O., Shen, Z., Guo, B., Shen, J., et al. (2012). A fluoroquinolone resistance associated mutation in gyrA affects DNA supercoiling in Campylobacter jejuni. Front. Cell. Inf. Microbio. 2:21. doi: 10.3389/fcimb.2012.00021

Hoang, K. V., Wang, Y., and Lin, J. (2012). Identification of genetic loci that contribute to Campylobacter resistance to fowlicidin-1, a chicken host defense peptide. Front. Cell. Inf. Microbio. 21:32. doi: 10.3389/fcimb.2012.00032

Johnson, E. M., Gaddy, J. A., and Cover, T. L. (2012). Alterations in Helicobacter pylori triggered by contact with gastric epithelial cells. Front. Cell. Inf. Microbio. 2:17. doi: 10.3389/fcimb.2012.00017

Kaakoush, N. O., and Mitchell, H. M. (2012). Campylobacter concisusa new player in intestinal disease. Front. Cell. Inf. Microbio. 2:4. doi: 10.3389/fcimb.2012.00004

Kim, I. J., and Blanke, S. R. (2012). Remodeling the host environment: modulation of the gastric epithelium by the Helicobacter pylori vacuolating toxin (VacA). Front. Cell. Inf. Microbio. 2:37. doi: 10.3389/fcimb.2012.00037

Liechti, G., and Goldberg, J. B. (2012). Outer membrane biogenesis in Escherichia coli, Neisseria meningitidis, and Helicobacter pylori: paradigm deviations in $H$. pylori. Front. Cell. Inf. Microbio. 2:29. doi: 10.3389/fcimb.2012.00029

Liu, H., Semino-Mora, C., and Dubois, A. (2012). Mechanism of H. pylori intracellular entry: an in vitro study. Front. Cell. Inf. Microbio. 2:13. doi: 10.3389/fcimb.2012.00013

Matos, R. G., Barria, C., Pobre, V., Andrade, J. M., and Arraiano, C. M. (2012). Exoribonucleases as modulators of virulence in pathogenic bacteria. Front. Cell. Inf. Microbio. 2:65. doi: 10.3389/fcimb.2012.00065
Miller, W. G., Chapman, M. H. Yee, E., On, S. L., McNulty, D. K., Lastovica, A. J., et al. (2012). Multilocus sequence typing methods for the emerging campylobacter species, C. hyointestinalis, C. lanienae, C. sputorum, C. concisus, and C. curvus. Front. Cell. Inf. Microbio. 2:45. doi: 10.3389/fcimb. 2012.00045

Neal-McKinney, J. M., and Konkel, M. E. (2012). The Campylobacter jejuni CiaC virulence protein is secreted from the flagellum and delivered to the cytosol of host cells. Front. Cell. Inf. Microbio. 2:31. doi: 10.3389/fcimb. 2012.00031

Noto, J. M., and Peek, R. M. (2012). The role of microRNAs in Helicobacter pylori pathogenesis and gastric carcinogenesis. Front. Cell. Inf. Microbio. 1:21. doi 10.3389/fcimb.2011.00021

Croinin, T. O., and Backert, S. (2012) Host epithelial cell invasion by Campylobacter jejuni: trigger or zipper mechanism? Front. Cell. Inf. Microbio. 2:25. doi: 10.3389/fcimb.2012.00025

Pernitzsch, S. R., and Sharma, C. M. (2012). Transcriptome complexity and riboregulation in the human pathogen Helicobacter pylori. Front. Cell. Inf. Microbio. 2:14. doi: 10.3389/fcimb.2012.00014

Plummer, P. J. (2012). LuxS and quorum-sensing in Campylobacter. Front. Cell. Inf. Microbio. 2:22. doi:10.3389/fcimb.2012.00022

Reid, D. W., Muyskens, J. B., Neal, J. T., Gaddini, G. W., Cho, L. Y., Wandler, A. M., et al. (2012). Identification of genetic modifiers of CagA-induced epithelial disruption in Drosophila.
Front. Cell. Inf. Microbio. 2:24. doi: 10.3389/fcimb.2012.00024

Sorensen, M. C., Van Alphen, L. B., Fodor, C., Crowley, S. M., Christensen, B. B., Szymanski, C. M., et al. (2012). Phase variable expression of capsular polysaccharide modifications allows Campylobacter jejuni to avoid bacteriophage infection in chickens. Front. Cell. Inf. Microbio. 2:11. doi: 10.3389/fcimb.2012.00011

Stahl, M., Butcher, J., and Stintzi, A. (2012). Nutrient acquisition and metabolism by Campylobacter jejuni. Front. Cell. Inf. Microbio. 2:5. doi: 10.3389/fcimb.2012.00005

Ta, L. H., Hansen, L. M., Sause, W. E., Shiva, O., Millstein, A., Ottemann, K. M., et al. (2012). Conserved transcriptional unit organization of the cag pathogenicity island among Helicobacter pylori Strains. Front. Cell. Inf. Microbio. 2:46. doi: 10.3389/fcimb.2012.00046

Received: 27 November 2012; accepted: 28 November 2012; published online: 19 December 2012.

Citation: Merrell DS and Stintzi A (2012) Research advances in the study of Campylobacter, Helicobacter, and Related Organisms. Front. Cell. Inf. Microbio. 2:159. doi: 10.3389/fcimb. 2012.00159

Copyright (C) 2012 Merrell and Stintzi. This is an open-access article distributed under the terms of the Creative Commons Attribution License, which permits use, distribution and reproduction in other forums, provided the original authors and source are credited and subject to any copyright notices concerning any third-party graphics etc. 